\title{
e-Migrinter
}

$20 \mid 2020$

Hospitalité et migration

\section{Quelques aperçus de mon vécu à Welcome Poitiers}

\section{Nelly Brimault}

\section{OpenEdition}

Journals

Édition électronique

URL : https://journals.openedition.org/e-migrinter/2233

DOI : $10.4000 /$ e-migrinter.2233

ISSN : 1961-9685

\section{Éditeur}

UMR 7301 - Migrinter

\section{Référence électronique}

Nelly Brimault, «Quelques aperçus de mon vécu à Welcome Poitiers », e-Migrinter [En ligne], 20 | 2020, mis en ligne le 23 juillet 2020, consulté le 20 mai 2021. URL : http://journals.openedition.org/emigrinter/2233; DOI : https://doi.org/10.4000/e-migrinter.2233

Ce document a été généré automatiquement le 20 mai 2021.

Tous droits réservés 


\title{
Quelques aperçus de mon vécu à Welcome Poitiers
}

\author{
Nelly Brimault
}

\section{Apporter sa pierre à l'édifice}

1 Tel le colibri qui apporte consciencieusement sa goutte d'eau pour éteindre l'incendie, j'apporte ma contribution à l'association Welcome de Poitiers.

2 J'ai pris contact avec cette association après une longue maturation de mes pensées quant à la situation des personnes migrantes et demandeuses d'asile (en France et dans le monde). C'était en février 2018. L'élément déclencheur a été ma rencontre avec Roukiata $^{1}$, émigrée originaire du Congo, hébergée depuis trois semaines chez Anna, une

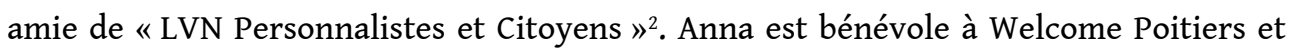
c'est dans ce cadre que pendant quatre semaines, elle offre le gîte et le couvert matin et soir à Roukiata. Ensuite Roukiata sera hébergée dans une autre famille du réseau, à Poitiers ou dans la Vienne, et ainsi de suite pendant six mois, durée maximale d'accueil dans le réseau Welcome.

3 Cette rencontre me toucha profondément, humainement. La belle et grande jeune femme qui partageait notre repas m'a émue par sa fragilité, sa tristesse, son désir de mère pour retrouver ses deux jeunes enfants restés au pays. Ses paroles et tout son corps exprimaient pour moi la souffrance, la lourdeur d'un destin subi trop lourd pour ses épaules. Cette femme avait l'âge de mes propres enfants. Elle était cultivée, soignée, attentionnée, désireuse de se rendre utile. Aimable au sens propre du terme. Sans mes formations professionnelles qui m'ont aidées à garder la distance nécessaire, j'aurais laissé libre cours à l'élan que je ressentais et l'aurais prise dans mes bras! L'attitude respectueuse et attentive de mon amie, son accueil chaleureux et simple, son humanité ont suscité mon admiration.

4 À partir de ce moment, j'ai décidé de faire « quelque chose ». Quoi ? Je n'en savais rien mais je ne pouvais plus rester spectatrice. J'ai décidé de consacrer une partie de mon 
temps de retraitée ainsi que de mon énergie à l'aide aux personnes migrantes. J'ai contacté Welcome et j'ai entraîné dans mon sillage mon compagnon Alain.

\section{L'association Welcome Poitiers}

5 Notre rencontre avec deux « responsables » de Welcome n'a pas tardé. Par un heureux hasard, l'association avait besoin d'étoffer ses bénévoles pour faire face à la demande d'accueil. Les différents postes à pourvoir m'ont été présentés: les familles d'accueil assurant l'hébergement, les «fileuses" responsables de la sélection des familles et, comme leur nom l'indique, du " filage ${ }^{3}$ de l'accueil et enfin les " tuteurs». Ce dernier rôle consiste à suivre, organiser et réaliser les déplacements d'une personne accueillie de famille en famille. Il se pratique en binôme.

Ces trois grands rôles se retrouvent dans le mode de fonctionnement de l'association, décrit sur le flyer de diffusion. Créée en 2016, l'association consiste en :

- Un réseau de familles qui se relaient pour offrir temporairement un hébergement à des demandeurs d'asile en situation régulière (six mois maximum).

- Un engagement ponctuel ou régulier à la mesure de chaque famille accueillante (qui assure hébergement, repas du soir et petit déjeuner).

- Une équipe de bénévoles impliqués qui accompagne chaque situation.

7 Notre rencontre avec les "responsables» de Welcome a rapidement été suivie d'une proposition de «filage " pour moi ; donc d'accompagnement d'une personne accueillie de famille en famille.

\section{Une expérience très personnelle et formatrice}

8 Accompagnée d'un bénévole « chevronné » j’ai rencontré Mahmoud, Guinéen de 30 ans. Si cette démarche peut paraître anodine, elle ne le fut pas pour moi... Nous avions pour mission de l'accueillir et de l'accompagner dans sa première famille d'accueil. Nous devions avec la famille et la personne accueillie lire les conditions d'accueil et nous assurer que pour les prochains jours le gîte, le couvert, les transports, le moyen de téléphoner soient assurés. Autant dire un rapide mais précis tour d'horizon de la situation. Comment ne pas étouffer cette personne qui vient juste d'être accueillie sous un flot de paroles, connaître un minimum de l'autre sans être intrusive? Enfin, ne pas oublier d'échanger les coordonnées, notamment le numéro des tuteurs qui font le lien entre les familles d'accueil et leurs hôtes pendant tout leur séjour. Le tuteur est un médiateur auquel chacun peut faire appel en cas de besoin. Des besoins très variés: achat d'une carte de téléphone, besoin d'un transport vers l'OFII ${ }^{4}$, ajustement au mode de vie de la famille, question de régime, respect du ramadan...

Pour cette première situation, j'ai bien apprécié d'être accompagnée par mon cotuteur. Personnellement je ne savais que dire du pays d'origine que je ne connaissais pas, et je n'osais pas questionner par crainte d'être intrusive et de paraitre très inculte. Mahmoud était lui-même quasiment muet, répondant très brièvement. D'où venait-il ? Comment était-il arrivé en France? Quels étaient ses projets? Ma tête fourmillait de questions que je n'ai pas posées. Heureusement la famille devait résoudre des questions pratiques, ce qui facilita le dialogue. Mahmoud était-il musulman? Mangerait-il du porc? Pourrait-il aller seul jusqu'à l'arrêt du bus? Avait-il des tickets? Avait-il une 
adresse e-mail ? Connaissait-il la banque alimentaire? Et le 115 ? et le Secours Catholique ? Et la PADA $^{5}$ ? Avait-il un plan de Poitiers ?... J'ai finalement simplement donné la parole, tendu une perche à Mahmoud afin qu'il exprime lui-même ses besoins.

\section{Un univers à découvrir... Les organismes d'aides aux migrants}

La bonne volonté c'est bien, mais cela ne suffit pas! Dès la première rencontre j'ai été confrontée à ce qui m'a semblé être un véritable jargon de spécialiste.

Bien sûr, en raison de mon ancienne profession (assistante sociale) je connaissais déjà la Banque alimentaire, le Secours Catholique, le Relais Georges Charbonnier ${ }^{6}$, et si j'avais entendu parler du « 115 », j'ignorais où il se trouvait, sa capacité d'accueil et les conditions d'hébergement.

Mahmoud ne percevant pas correctement son $" A D A »^{7}$, on m'a demandé de contacter « l'OFII ». Et si j'avais un problème? Je n'avais qu'à contacter la «PADA» locale ... Autant dire pour moi des sigles inconnus correspondants à des organismes inconnus...

13 Devant tant d'inconnu je me suis demandée si j'allais être à la hauteur et si ma motivation allait être suffisante pour apprendre encore et encore, plutôt que de m'enfoncer dans mon confortable fauteuil de retraitée! Mais c'était sans compter sur Baptiste, Hélène et Myriam! Autant d'interlocuteurs stimulants qui m'ont expliqué et encouragée pour aborder la complexité du système. Oui c'est compliqué! Non, à Poitiers il n'y a pas pléthore d'organismes. Du fait de mon désir de savoir, de mes précédents acquis et de ma mobilité, j'aurai tôt fait de me familiariser. Bref ! En quelque sorte je me retrouvais « en stage ", à 68 ans et j'ai trouvé cela plutôt vivifiant !

\section{Les familles d'accueil et la personne accueillie}

14 À chaque accueil, c'est une savante alchimie qui se crée autours d'un contrat écrit, lu, et accepté, mais surtout grâce aux relations humaines réciproquement respectueuses. D'une famille à l'autre, j'ai accompagné Mahmoud chez François et Sylvie, propriétaires d'une grande maison à la campagne et entourés d'animaux et de champs, puis chez Lydie, retraitée, vivant seule dans une petite maison de centre-ville avec jardinet de $9 \mathrm{~m} 2$. Chez les uns, pas de magasin à moins de dix kilomètres. Chez l'autre, le Relais Georges Charbonnier et la Banque Alimentaire à proximité. Chez Lydie, Mahmoud devra quitter la maison chaque matin comme prévu dans le contrat. Chez François et Sylvie, il pourra rester toute la journée et participer aux travaux de la ferme ... Quelle adaptation nécessaire!

15 Le tuteur permet à chaque famille d'exposer son mode d'accueil afin que la personne accueillie en ait bien conscience. Le tuteur peut expliquer les habitudes, faire des mises au point. En horticulture «le tuteur » est un bâtonnet qui sert à soutenir ou redresser une plante. Je suis amoureuse des plantes, aussi c'est cette image qui m'est venue à l'esprit pour expliquer à Théodoro qu'il devait rectifier son attitude dans sa famille d'accueil. Il devait apprendre à respecter les horaires de repas et ne pas imposer ses programmes de télévision à la personne qui l'accueillait. La connaissance, puis l'adaptation aux habitudes quotidiennes des familles accueillantes passe par des apprentissages. Le tuteur peut avoir ce rôle de transmission, d'information auprès des 
« accueillis ». Rôle parfois ingrat mais nécessaire. En effet, les migrants que nous aidons ne sont pas des enfants mais des adultes et certains ignorent parfois nos habitudes de vie ou nos lois. Le tuteur doit donc avoir le tact et le respect qui s'imposent, tout en essayant de faire passer des messages parfois compliqués afin que l'accueil se déroule pour le mieux.

16 J'ai observé un point commun à toutes les familles. Je suis étonnée et émerveillée de la qualité des accueils, de la souplesse, de la générosité et de l'ouverture d'esprit des familles actives dans ce réseau à Welcome. J'ai découvert une belle face de l'humanité, capable de partager son toit, sa nourriture, voire sa salle de bain ou son véhicule, de s'adapter en cas de besoin, de consacrer une partie de son temps. J'ai plus d'une fois ressenti de l'émotion lors des échanges de fin de séjour. Nombreuses sont les familles qui ont remercié les accueillis pour la qualité des échanges, chacun s'accordant à reconnaître qu'ils y avaient gagné autant que donné ! Que d'embrassades, d'adresses et d'au revoir échangés !

17 J'ai trouvé ces expériences très réconfortantes et totalement en opposition avec un discours raciste ou de rejet de l'étranger. Cela m'a fait du bien.

\section{Et « pan sur le bec $»^{8}$ ! Ou comment faire des bourdes par ignorance!}

Quand Sanza, accueillie à Welcome, est arrivé avec 35 minutes de retard à notre rendezvous, cela m'a passablement énervée, pointilleuse que je suis quant au respect des horaires! J'ai manifesté mon agacement et expliqué qu'il convenait d'être ponctuel, sans quoi cela risquait de le mettre en difficulté. Quand la famille d'accueil m'a avisée qu'elle était gênée par le fait que Sanza arrivait en retard pour les repas ou ne venait pas du tout, mon co-tuteur et moi-même avons invité Sanza pour faire le point. Une bonne heure d'échanges m'aura été nécessaire pour que je réalise à quel point mon attitude faisait fi des habitudes de Sanza acquises dans son pays. Arrivé en France récemment, il n'avait jamais travaillé pour un employeur avec des horaires précis, il mangeait une fois par jour à l'heure de son choix dans sa famille et n'avait aucune nécessité de prévenir. J'ai pris conscience que les réalités de deux mondes se rencontraient, se confrontaient. Je me suis sentie très confuse et soucieuse d'avoir rajouté des reproches au vécu suffisamment difficile.

19 J'avais bien du chemin à parcourir, mais Sanza aussi! Il est dans l'absolue nécessité de comprendre le fonctionnement de notre société. Je n'en suis qu'un témoin! Avec Sanza, nous avons mis des mots sur cette expérience qui, j'espère, lui a permis d'avancer.

\section{Une expérience dérangeante ! Ou croiser l'itinéraire d'un migrant}

Si la rencontre avec une personne migrante peut paraître banale, elle ne l'est pas du tout pour moi. Croiser le regard d'un SDF dans la rue est difficile, mais partager un moment avec une personne réfugiée et inconnue n'est pas simple! Dans le cadre de Welcome, et pour un premier contact, je sais déjà que je vais rencontrer une personne sans logement, mais j'ignore comment et d'où elle est arrivée, quel est son parcours, ses 
habitudes, ses besoins. Lors de mes premières "prises en charge ", j'avais quasiment des maux de tête du fait des questions qui tournaient en boucle dans ma tête.

21 Je suis confortablement installée dans ma vie de retraitée, je possède ma voiture, ma maison, mes grands-enfants sont autonomes. Quel décalage! J'ai souvent le sentiment de ne pas vivre sur la même planète que les accueillis. Je ne connais que rarement leur parcours, sauf quand ils m'en parlent spontanément. Je l'imagine plein de périls et d'embûches. Je crois que ceux qui parviennent jusqu'à nous sont forts et extrêmement motivés mais je crains le moment de leur départ, après six mois d'accueil à Welcome. Le retour à la rue me semble impensable! D'ailleurs, qu'adviennent-ils d'eux ? Certains sont admis en $\mathrm{CADA}^{9}$ et d'autres rejoignent des amis qu'ils se sont faits au cours de leurs mois à Poitiers.

Mon action à Welcome me semble tour à tour dérisoire, puis utile, et finalement suffisamment positive à mes yeux pour que je continue. L'accueil et la générosité des familles envers les accueillis me semble admirable et me réconforte. Le fait que Mandou ait obtenu son statut de réfugié et suive une formation en alternance dans une entreprise est carrément une source de joie et de motivation pour moi. Alors oui, je continue d'apporter ma brique à l'édifice collectif Welcome 86 dont je partage les valeurs.

\section{NOTES}

1. Dans un souci d'anonymat, j'ai choisi des noms d'emprunt.

2. La Vie Nouvelle, Personnalistes et Citoyens : association nationale dont je suis coprésidente pour le groupe de la Vienne. Mouvement d'éducation populaire.

3. Le filage est le nom donné à l'organisation de la succession des familles d'accueil pour une personne accueillie sur une période de six mois.

4. Office français de l'immigration et de l'intégration.

5. Plateforme d'Accueil des Demandeurs d'Asile.

6. Centre d'accueil qui assure une prise en charge globale des publics les plus fragiles (repas, soins médicaux, etc.).

7. Allocation pour demandeurs d'asile.

8. «Pan sur le bec » est une expression du Canard enchaîné dans la rubrique du même nom, où le Canard reconnaît ses erreurs.

9. Centre d'accueil de demandeurs d'asile. 
INDEX

Index géographique : France, Vienne

Mots-clés : associations, demandeurs d'asile, accueil, vie quotidienne, témoignage

\section{AUTEUR}

\section{NELLY BRIMAULT}

68 ans, assistante sociale retraitée. Bénévole à l'association Welcome de Poitiers depuis 15 mois nellybrimault@wanadoo.fr 